\title{
Isolated Unilateral Absence of Pulmonary Artery (UAPA) in the Adult: Between Simple Cough and Intensive Care
}

\author{
Jacob FJ*, Vogt FM, Stahlberg E, Oechtering TH, Anton S, Planert M, Schierholz S, Barkhausen J and Frydrychowicz A
}

Clinic for Radiology and Nuclear Medicine, University Hospital of Schleswig Holstein, Campus Lubeck, Lubeck, Germany

\begin{abstract}
Unilateral absence of pulmonary artery (UAPA) is a rare congenital condition occurring isolated or in conjunction with other congenital heart defects (CHD). While none-isolated UAPA is mostly detected early in life, detection of isolated UAPA is often delayed due to diverse and unspecific symptoms. While there is consensus on the therapeutic strategy in young symptomatic patients, the incidental finding of UAPA in young or adult asymptomatic patients is a matter of on-going debate and limited evidence. Here, we will present two previously unpublished, illustrative cases with different extent of symptom severity and discuss the difficulties in determining an appropriate therapeutic strategy. Along with the cases, we will review the literature, present typical symptoms and possible pitfalls, and provide an overview of therapeutic options with a focus on the adult patient.
\end{abstract}

Keywords: Isolated unilateral absence of pulmonary artery (UAPA); Pulmonary hypertension; Pneumonectomy; Revascularization; Hemoptysis

\section{Introduction}

The origin of UAPA, the unilateral absence of the pulmonary artery, is attributed to the fetal period. UAPA is thought to result from embryonic involution of either the left or the right proximal portion of the sixth aortic arch which usually transforms into the pulmonary artery. At that time, the lung buds are supplied through the bronchial arteries and are thus independent of the pulmonary arteries. Thus, blood supply to the affected lung is maintained despite the lack of a pulmonary artery perfusion due to systemic collaterals and bronchial arteries that are non-obliterating. This compensatory mechanism will later be one of the potential sources of complications when haemorrhages through bronchial feeders and collaterals may occur. The prevalence of UAPA has been described to be on the order of 1: 100,000 and is associated with cardiac abnormalities in about $45 \%[1,2]$. While sinistral or leftsided UAPA is less common (37\%) than dextral or right-sided UAPA $(67 \%)$, it is associated with an increased rate of congenital heart defects (CHD) on the order of 75\% [3]. Among the associated CHDs, tetralogy of Fallot, septal defects, aortic coarctation, subvalvular aortic stenosis, transposition of the great arteries, and scimitar syndrome have been described [1]. If associated with other CHDs, UAPA is typically diagnosed during the diagnostic CHD workup in childhood and can be corrected surgically by reconnecting the pulmonary trunc and the hilar artery [2]. In case of isolated UAPA, 15-27\% of patients remain asymptomic $[1,4]$. Symptomatic patients often present with unspecific symptoms such as chest pain, dyspnea, exercise limitations, pleural effusion, recurrent pulmonary infections, haemoptysis, or pulmonary hypertension $(\mathrm{PH})$. Therefore, isolated UAPA is typically a coincidental finding during diagnostic testing [1]. Symptomatic adult UAPA is rare and typically, the diagnosis is being established later in life after narrowing in on heterogeneous symptoms. Hence, therapeutic guidelines are missing. Therefore, this review and presentation of two cases with different scope aims at summarizing potential pitfalls during diagnosis and therapeutic decision-making in isolated UAPA.

\section{Case Series}

Patient 1, a 36 year-old mother-of-three was admitted to the emergency department of our tertiary care medical center with haemoptysis after episodes of recurrent pulmonary infections. Case history revealed a similar episode about 7 years ago during pregnancy, treated in a primary hospital. Due to pregnancy, no in-depth diagnostic workup was performed and she was lost to post-partum follow-up. The recent diagnostic workup included an initial chest X-ray depicting diffuse patchy right-sided infiltrations, normal left lung and heart, but an amputated right hilum (Figure 1). Cardiac auscultation as well as electrocardiogram (ECG) was normal. Bronchoscopy revealed diffuse right bronchial bleeding, most pronounced in segments 2 and 3 . Initial therapy included epinephrine-lavage of both upper and lower right lobes. During intervention the patient was intubated because of an oxygen desaturation due to massive pulmonary bleeding. After cardiorespiratory stabilization on the intensive care unit a computed tomography (CT) of the pulmonary arteries was performed revealing right-sided, isolated UAPA with multiple systemic feeder arteries (Figure 2). Alveolar infiltrates concordant with parenchymal bleeding, bronchiectasis, and fibrosis were seen as well. As seen in Figure 3, subsequent aortic angiograms by DSA confirmed multiple systemic feeder arteries without proof of bleeding source. After 3 days she was extubated, the primary antibiotic regime was de-escalated and she was transferred to a normal ward. Further diagnostic workup included a 6-minutes-walking test showing a slight blood pressure increase, so a low dose beta blocker therapy has been started. Right heart catheter measurements were unsuspicious with no intracavitary and pulmonary artery pressures elevation. Transthoracic echocardiography (TTE) was normal. In preparation of a potential surgical approach, a lung ventilation/perfusion scan was performed yielding absence of right pulmonary perfusion, but only slightly decreased ventilation (Figure 4). After 10 days on normal ward, her general conditions improved, antibiotic therapy has been stopped and no further episodes of haemoptysis appeared so that she was discharged and follow-up in an outpatient clinic. However, two weeks later, recurrent mild haemoptysis following hard physical labour led to re-admission. Reassessment and

${ }^{*}$ Corresponding author: Fabian Jacob, Clinic for Radiology and Nuclear Medicine, University Hospital of Schleswig Holstein, Campus Lubeck Lubeck, Germany, Phone: +49 451 500-21 29; Fax: +49 451 500-64 97; E-mail: fabian.jacob@uksh.de

Received May 21, 2018; Accepted May 25, 2018; Published May 31, 2018

Citation: Jacob FJ, Vogt FM, Stahlberg E, Oechtering TH, Anton S, et al. (2018) Isolated Unilateral Absence of Pulmonary Artery (UAPA) in the Adult: Between Simple Cough and Intensive Care. J Pulm Respir Med 8: 457. doi: 10.4172/2161105X.1000457

Copyright: @ 2018 Jacob FJ, et al. This is an open-access article distributed under the terms of the Creative Commons Attribution License, which permits unrestricted use, distribution, and reproduction in any medium, provided the original author and source are credited. 


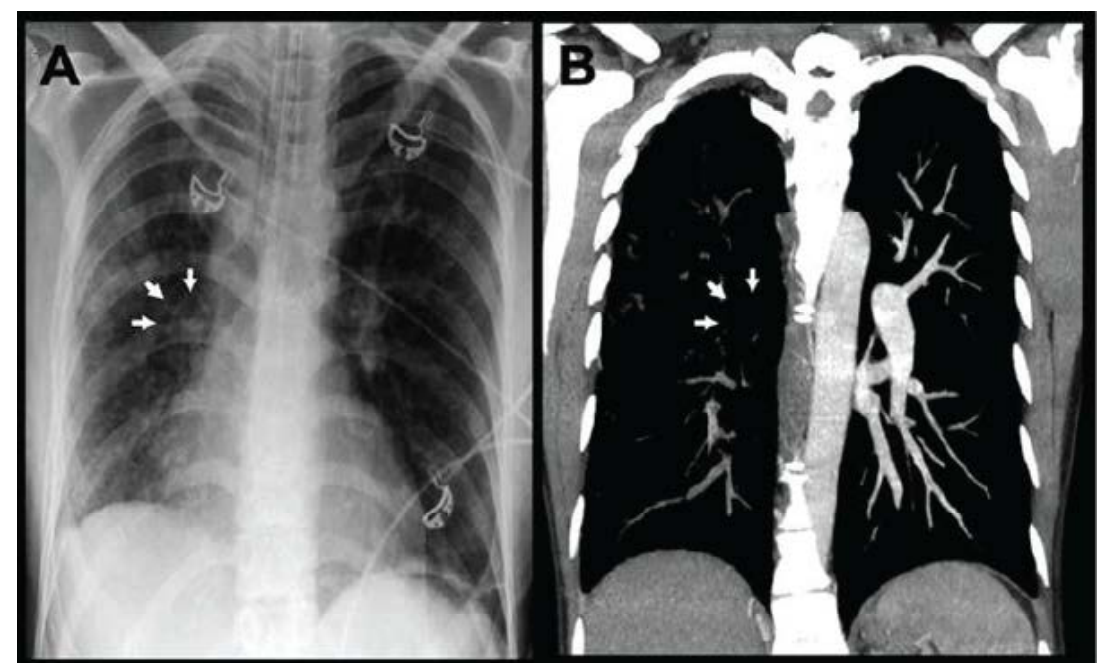

Figure 1: Chest radiograph (A) demonstrating right-sided patchy infiltrations, right hilar vasculature was absent (arrows), and pericardial blurring due to middle lobe bronchiectasis and dystelectasis. Coronally reformatted maximum intensity projection of computed tomography (B) confirmed the absence of the right pulmonary artery (solid arrows).

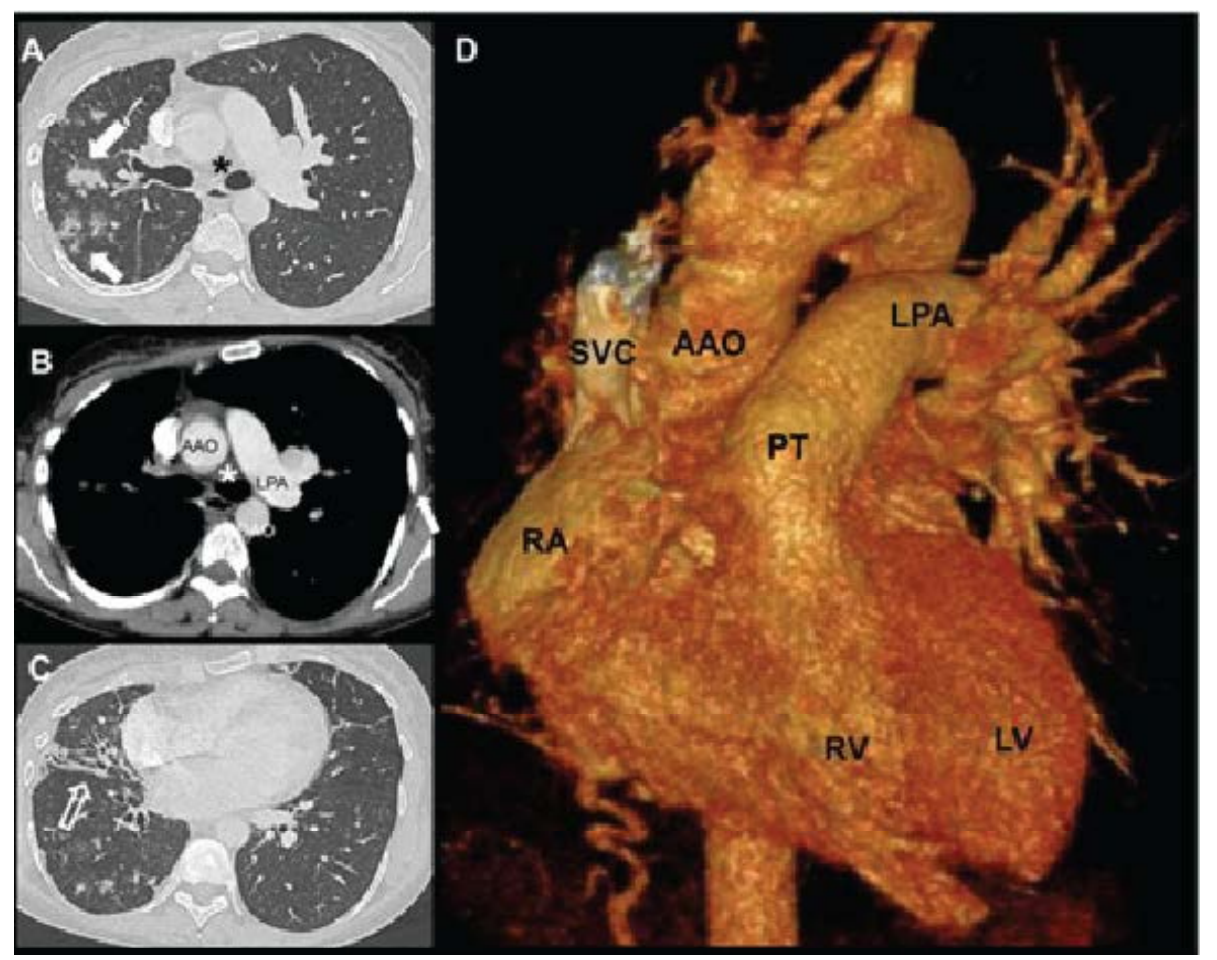

Figure 2: Absence of the right pulmonary artery (A, B; asterisks) was confirmed by standard chest computed tomography performed for diagnosis of pulmonary hemorrhage. No other cardiac anomalies were seen. Patchy infiltrates ( $\mathrm{A}$; solid arrows) indicated parenchymal bleeding. Bronchiectasis (C open arrow) appeared predominately in the middle lobe. The 3D volume rendered reconstruction (D) illustrated the absence of the right pulmonary artery. $\mathrm{AAO}=$ ascending aorta, $\mathrm{LPA}=$ left pulmonary artery, $\mathrm{PT}=$ pulmonary trunk, $\mathrm{SVC}=$ superior vena cava, $\mathrm{RA}=$ right atrium, $\mathrm{RV}=$ right ventricle, $\mathrm{LV}=$ left ventricle.

preparations for pneumonectomy were discussed, but further medical treatment was refused by the patient.

Patient 2, a 63 year-old male with permanent atrial fibrillation, fusiform holosystolic murmur across the aortic valve and ECG changes (inverted T wave in II, aVL and V6) was transferred from domesticity to our hospital due to angina pectoris complaints. Known previous illnesses included episodes of pulmonary infections, type-2-diabetes and chronic renal failure stage II. Initial coronary angiography excluded coronary artery disease but confirmed an aortic valve stenosis III ${ }^{\circ}$ and an ascending aortic aneurysm. Preoperative CT revealed a right-sided, isolated UAPA with systemic feeder arteries from the descending aorta and the inferior phrenic artery (Figure 5). The right lung volume was reduced, probably due to a lesser extent of perfusion, and lung parenchyma displayed scarred-fibrotic alterations as a hint for past 
Citation: Jacob FJ, Vogt FM, Stahlberg E, Oechtering TH, Anton S, et al. (2018) Isolated Unilateral Absence of Pulmonary Artery (UAPA) in the Adult: Between Simple Cough and Intensive Care. J Pulm Respir Med 8: 457. doi: 10.4172/2161-105X.1000457

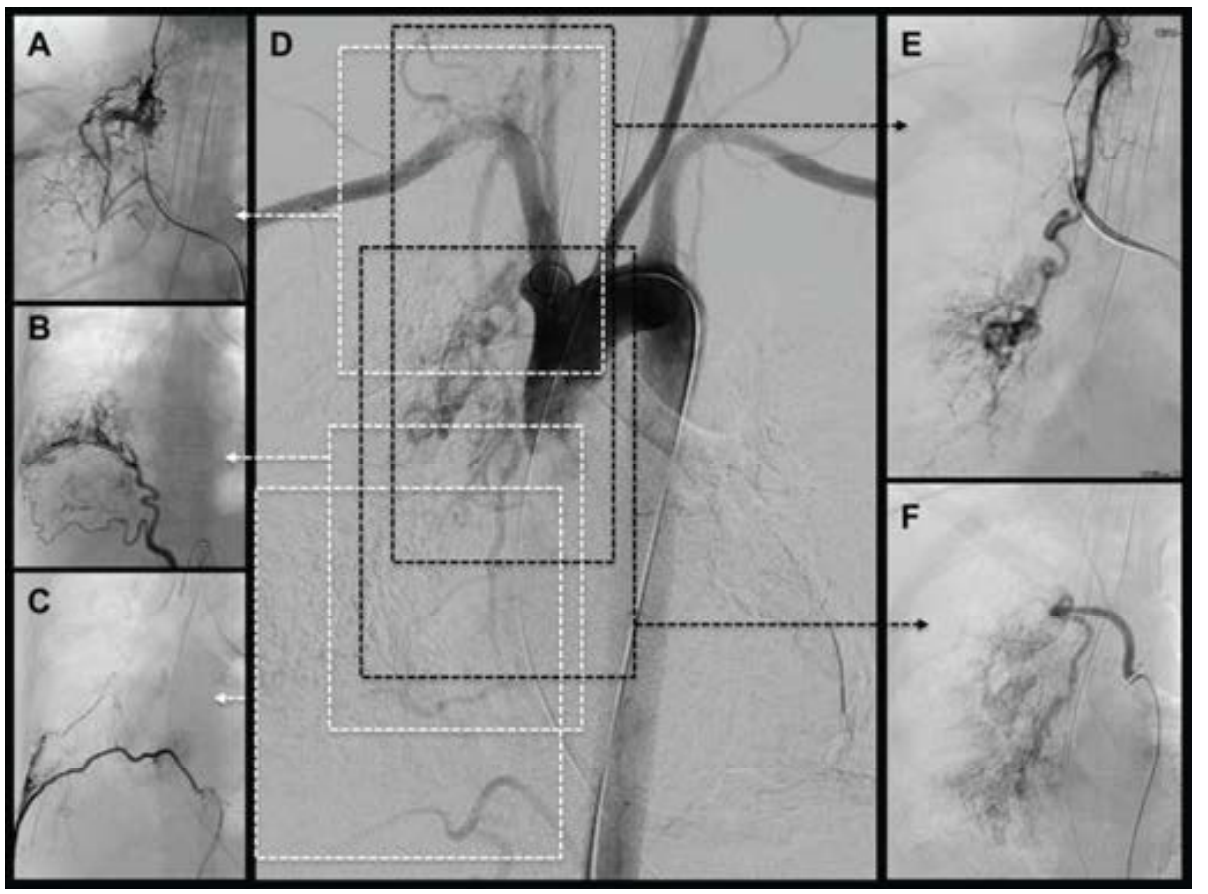

Figure 3: Selective digital subtraction angiography (DSA) of the thoracic arteries demonstrated multiple feeder arteries of the right lung. Arteries arising of the costocervical trunk (A), inferior phrenic artery (B), multiple intercostal arteries (exemplary right lower intercostal artery shown in C), subclavian artery (E) and the bronchial arteries $(\mathrm{F})$ contributed to the collateral blood supply of the right lung.

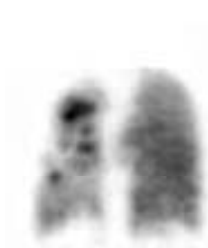

R V L

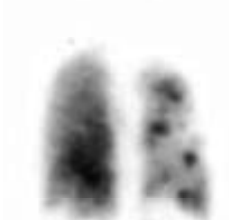

L D R

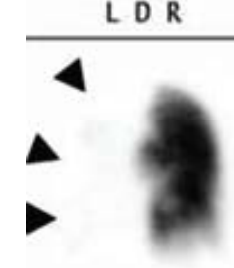

R V L

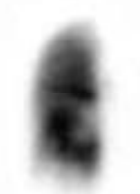

L D R

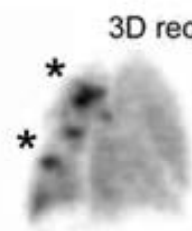

RAO

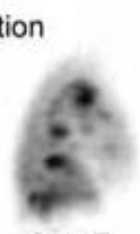

R LAT

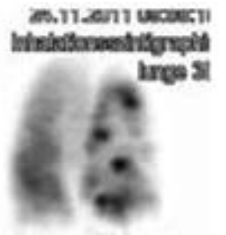

RPO

Inhalation
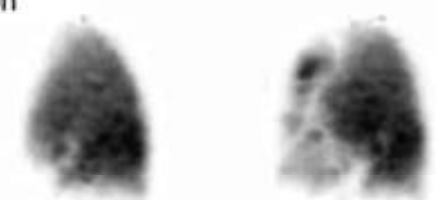

L LAT

LAO

LPO

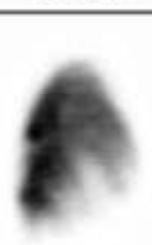

R LAT

RPO
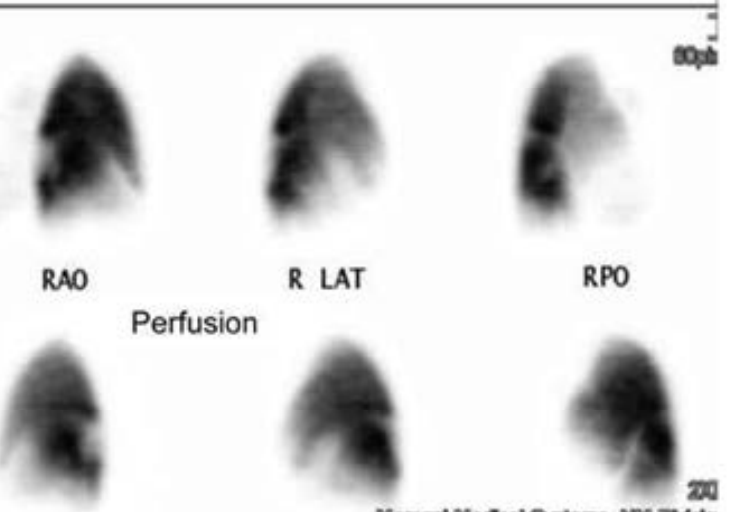

Perfusion

LPO

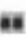

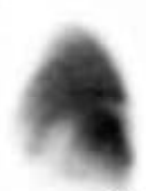

L LAT

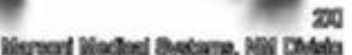

LAO No nat

Figure 4: Ventilation/perfusion scintigraphy revealed preserved ventilation while no pulmonary arterial perfusion of the right lung (arrowheads) was observed. Ventilation 'hot spots' (asterisk), congruent with massive bronchiectasis were also detected and matched CT-findings. 


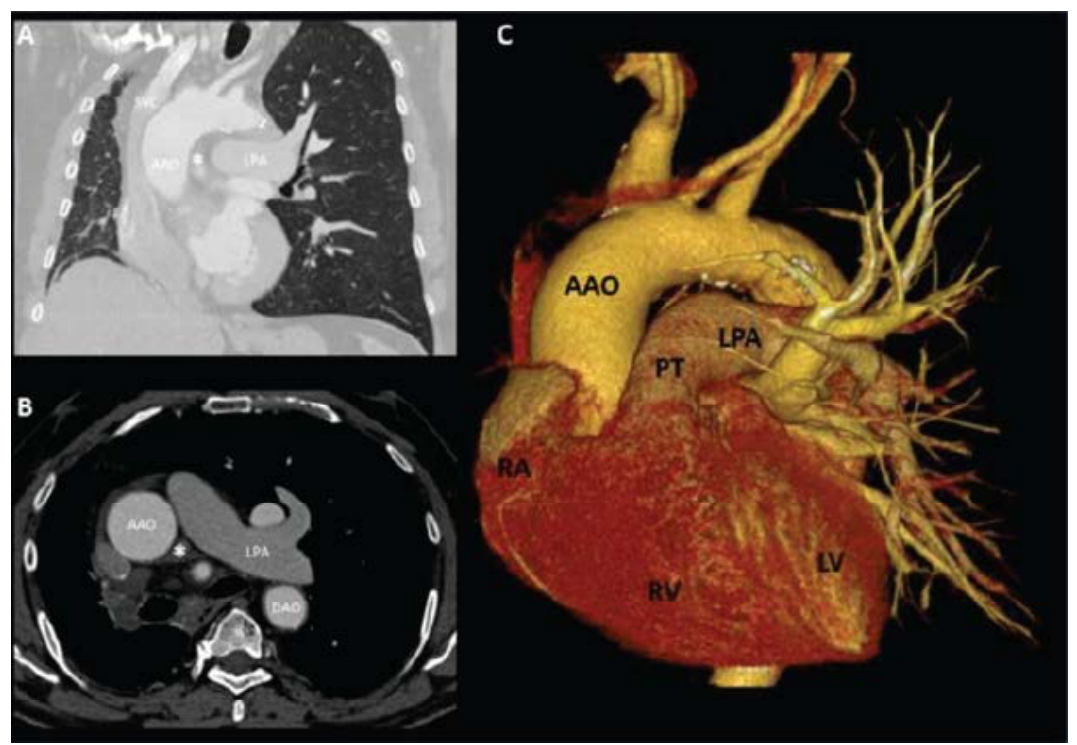

Figure 5: Absence of the right pulmonary artery (A, B; asterisks) was confirmed by standard chest computed tomography performed for aortic valve replacement. No other cardiac anomalies were seen. The $3 D$ volume rendered reconstruction $(C)$ illustrated the absence of the right pulmonary artery. $\mathrm{AAO}=$ ascending aorta, $\mathrm{LPA}=$ left pulmonary artery, $\mathrm{PT}=$ pulmonary trunk, $\mathrm{SVC}=$ superior vena cava, $\mathrm{RA}=$ right atrium, $\mathrm{RV}=$ right ventricle, $\mathrm{LV}=\mathrm{left}$ ventricle.

pulmonary infections. The elective surgical aortic valve replacement including resection of the left atrial auricle per local standard was successfully performed without complications. Post-operative TTE showed a good systolic left ventricular function and no signs of $\mathrm{PH}$. Repeat follow-up TTE was recommended.

\section{Pathophysiologic and diagnostic considerations}

If not associated with CHD, UAPA is often diagnosed coincidentally during late adulthood and can retrospectively be attributed to previous unspecific symptoms as exemplified by patient 2 . Although the presented symptoms match the diagnosis, they are far from being specific and not to speak of being pathognomonic. Related to the hemodynamic peculiarities of UAPA, symptoms can be induced or aggravated by hard physical labour, high-altitude or by pregnancy with its increased blood volume and hyperemia as case 1 suggests [1].

As part of the clinical examination, cardiac auscultation can reveal a systolic murmur across the pulmonary outflow tract, especially in case of accompanying pulmonary hypertension. During pulmonary examination, UAPA can be associated with diminished breathing sounds, course crackles and a hypersonoric resonance on percussion. Also, a restrictive pattern can be observed in pulmonary function test $[5,6]$. Similarly, chest radiograph can vary depending on ipsilateral lung inflation and perfusion. Typically, the affected lung is smaller due to atelectasis causing a mediastinal shift.

Also a missing hilar vasculature can be observed [7]. Yet, these signs are not mandatory and if they are present, they are unspecific and can easily be missed or misinterpreted. As displayed by Case 2 the diagnose UAPA was made after carried out emergency diagnostics and coronary angiography throughout the preoperative CT.

If not established coincidentally, the working hypothesis of UAPA will typically be further evaluated or diagnosed by cross-sectional imaging. Both, CT and magnetic resonance imaging (MRI) are able to address the question. In about $40 \%$ of cases, UAPA is associated with recurrent pulmonary infections and signs thereof [1]. The propensity towards infections is a result of diminished blood supply, followed by bronchoconstriction and mucous plugging which in turn can lead to chronic infections and result in fibrosis and bronchiectasis [1,7]. Thus lung parenchyma changes appear regularly in case of UAPA the CT is preferable as first cross-sectional imaging, whereas MRI should be preserved for younger patients and precise cardiovascular questions.

In cases of acute pulmonary haemorrhage as the initial symptom, bronchoscopy may be the method of choice during the acute setting giving an indication to the diagnosis. As aforementioned, haemorrhages can manifest when systemic arterial collaterals or bronchial arterials feeders rupture, especially in situations with increased systemic perfusion such as in hard labour, exercise, or pregnancy. Alternatively, there have been haemorrhages due to pulmonary hypertension that can affect both lungs or even the contralateral lung with pulmonary arterial supply [8]. However, even in the acute situation, high spatial resolution arterially contrast-enhanced computed tomography angiography (ceCTA) will often be initially performed. On basis of ceCTA, the UAPA, collateral arteries and bronchial arterial feeders can be detected and haemorrhages confirmed and further therapy guided. Of note, even coronary arteries can be the culprit vessel [3]

In 2010, Koga et al. revealed an association of isolated UAPA with pulmonaryhypertension $(\mathrm{PH})$ in $32 \%$ ( $\geq 20$ years of age, mean pulmonary artery pressure (mPAP) $54 \pm 16 \mathrm{mmHg}$ ). In UAPA, $\mathrm{PH}$ is the result of a single lung perfusion causing elevated sheer stress in the contralateral pulmonary arteries (PA) leading to chronic vasoconstriction followed by vascular remodelling. This association and the $\mathrm{PH}$ mortality rate of up to $>10 \%$ per year (European Society of Cardiology Guidelines 2015) emphasize the critical role of $\mathrm{PH}$ in long-term survival on the one hand and stresses the importance of early diagnosis and follow-up on the other. Transthoracic echocardiography (TTE) is recommended by the European Society of Cardiology for the initial diagnoses and regular follow-up examinations for $\mathrm{PH}$ and furthermore can be the first step towards excluding potentially associated cardiovascular anomalies [6]. While TTE is easy to perform and easily available, there are limitations to transesophageal echocardiography (TEE) that lends itself well to confirming the diagnosis of UAPA. 
Invasive diagnostic approaches play a decreasing role in the diagnostic strategy of patients with UAPA. If UAPA associated PH is suggested by TEE, a right heart catheter (RHC) should be performed remaining the diagnostic gold standard. However, with the vast availability of high-resolution, high-pitch ceCTA, angiographic catheter exams are not the method of choice for the sole purpose of diagnosing UAPA anymore. More dominantly, angiography is the method of choice for correctly locating sources of bleeding with the unmet potential of immediate embolization of the bleeding source.

\section{Clinical Suggestions}

As aforementioned, diagnosing patients with isolated UAPA can be challenging. However, based on different extent of disease severity and urgency ranging from asymptomatic patients such as in case 2 to life threatening symptoms as seen in case 1 , therapeutic considerations can be even more demanding. Beside these the patient's age will be an important factor in clinical decision making. Due to the sparsity of data and experience, little official recommendations or guidelines are available.

For symptomatic neonates and young children, surgical revascularization is an accepted therapeutic approach under the assumption that close to normal lung development can be achieved by restoring anatomically improved blood supply to the affected lung thus normalizing $\mathrm{PH}$ if present. The lack of non-invasive treatment options in case of acute onset of severe symptoms (CHD or increased $\mathrm{PH}$ ) legitimize an operative procedure $[2,4,7,9]$.

However, in case of early diagnosis of a symptom-free patient no guidelines exist. For isolated UAPA in the asymptomatic adult only suggestions based on the actual literature can be found. In cases without evidence of cardiopulmonary dysfunction, $\mathrm{PH}$, and other symptoms, no treatment is needed, but regular medical follow-up including TTE and blood pressure control is recommended [10]. Therapeutic options for the symptomatic adult patient include conservative approaches including changes in life-style or drug therapy and procedures of increasing invasiveness comprising of bronchoscopic and/or interventional procedures and surgical procedures including pneumonectomy, revascularization, or heart-lung-transplantation. In any case, patients should be evaluated for factors that can trigger or worsen the symptoms and can easily be avoided such as smoking, high altitude, hard physical labour, or are self-limiting such as pregnancy. In the following, we will summarize therapeutic options in light of specific symptoms. Due to their inherent peculiarities, we will put emphasis on the above-mentioned topics haemoptysis, pregnancy and pulmonary hypertension.

Recurrent episodes of haemoptysis can be self-limiting over many years, but can also be life-threatening if associated with relevant blood loss, alveolar infiltration and loss of oxygen exchange possibilities [7]. Patient should be aware of possible triggers and pharmacological systemic and/or pulmonary blood pressure control should be implemented. If massive bleeding cannot be stopped by bronchoscopic intervention, indication for embolization procedure is an individual and questionable decision. Data from Koga et al. in included 13 patients with hemoptysis, 6 with an identified active bleeding source in DSA. Out of those only one patient was successfully occluded due to massive collateralization, emphasizing the procedure difficulties in patients with UAPA. Even if embolization is successful long-term recurrence rates were up to $25 \%$ due to the collateralization [7]. This debatable outcome, which, as a single center result, is likely not entirely representative, points out the necessity of strict indication especially due to potential severe complications like pulmonary infarction and medullary artery embolization [11]. In case of unstoppable massive bleeding or severe recurrent haemoptysis of the ipsilateral side of patients with UAPA, a lobe- or pneumonectomy can be the last and ultimate therapeutic resort. To keep blood loss in scheduled surgical procedures minimal, a preoperative embolization is a widely accepted approach [6,12-14]. In our search of the literature, we compiled 22 cases of documented pneumonectomy in the adult from 1962 up to 2016 with one reported death due to excessive blood loss [1-3,4,6,10,12,15-17]. In this case no preoperative embolization had been performed, which could have been life-saving, so the expectable mortality-rate for pneumonectomy is most likely less than 5\%. In all 21 patients no further episodes of haemoptysis were reported after pneumonectomy in short-term followed up, but long-term follow up data are missing [3,6,12,14,15].

Pulmonary infections in case of UAPA do not need a specialized treatment strategy keeping the altered pathogen spectrum in case of bronchiectasis and the occurrence of pulmonary fibrosis in mind. In case of severe or therapeutic resistant infection and destruction of lung parenchyma, a lobe-or pneumonectomy can be considered, if intensive conservative treatment fails and operative risk is justifiable. Usually patients are symptom-free afterwards and in individual cases even a higher physical capacity is reported [15]. The modus operandi and the risk factors for pneumonectomy are the same as mentioned above.

The high association of UAPA and PH is probably the most severe complication, potentially resulting in right heart failure, PA aneurysmal dissection or rupture [18]. Whereas recurrent infections and haemoptysis can be cured by pneumonectomy, there is no curative treatment for $\mathrm{PH}$ associated UAPA except for heart and lung transplantation. Early diagnosis of $\mathrm{PH}$ in the adult through regular TTE is important to monitor remodelling that should be prevented or slowed by behavioural and pharmacological treatment. Supportive oxygen supply has shown a positive effect as well in patients with UAPA $[19,20]$.

Even if no randomized studies exist, the perceived pathomechanism and individual reports of successful pharmacological treatment $[19,21]$ suggest that UAPA associated PH should be treated like pulmonary arterial hypertension (PAH). The ESC/ERA guidelines from 2015 recommend a basal follow up every 3-6 month including routine medical check-up, ECG and 6-minute walk test and extended followup including an additional TTE every 6-12 month. Pharmacological treatment regime depends on the severity of $\mathrm{PH}$ and response should be evaluated 3-4 months after starting or changing therapy. If $\mathrm{PAH}$ adjustment is insufficient a higher dosage, another therapeutic agent or a triple therapy would be the next step.

Whereas revascularization procedure of neonates and young infant with UAPA and PH and/or CHD became the gold standard, there is still a therapeutic twilight zone for symptom-free infants and (a-) symptomatic adults. Probably the most encouraging study for younger end-stage patients with UAPA and PAH was presented by Bockeria et al. [2]. Next to neonates and young children the study $(\mathrm{N}=37)$ included 4 patients $>18$ years of age and 25 patients from 1-18 years of age demonstrating that operation in older infants or even adults is feasible, if a pulmonary artery at the hilum is present. In conjunction with the observation of Koga et al. and Atik et al. [4,9]. In 2006, reconstruction procedure can improve $\mathrm{PH}$, presenting another treatment option after conservative medical treatment, before heart-lung transplantation. The high association of $\mathrm{PH}$ of nearly one-third (patients $>20$ years of age) in isolated UAPA emphasizes the dilemma between early operating of a symptom-free infant versus late onset of $\mathrm{PH}$ with probably lifethreatening consequences. Revascularization in symptomatic neonates 
and young children has shown low in-hospital-mortality rate [2,9] with improvement of physical fitness and reduction of $\mathrm{PH}$, if present [9]. This supports the widely believed suggestion that early revascularization helps developing lung vasculature, offering a near to normal lung development. In spite of these encouraging results an operation of an asymptomatic infant could prevent the onset of $\mathrm{PH}$ - beside the other symptoms, but still is a hard decision to make. A preventive operation could also be taken into account under the perspective of a later volitional pregnancy.

Pregnancy with its physiological changes in blood volume and composition can unmask UAPA as indicated by the case history of the herein presented female patient. Simple dyspnea or exercise limitation in pregnant patients with isolated UAPA has to be differentiated from the same symptoms caused by $\mathrm{PH}$. At the end of gestation, blood volume increases to $40-100 \%$ above the pre-pregnancy baseline, whereas red cell mass only increases to $25 \%$ resulting in physiological anaemia [22]. Especially during labour and postpartum these changes result in strain for the right heart. If additional sympathetic stimulation raises the afterload or transfusion and venous return from uterine contraction increase the preload, pre-existing $\mathrm{PH}$ may be aggravated and lead to right heart failure [23]. While there is sparsity of data in UAPA-patients with $\mathrm{PH}$, mortality rates in pregnant $\mathrm{PH}$ patients without UAPA range from $12 \%$ to $56 \%$ [24-26].

To the best of our knowledge there is no randomized study data on mortality rates in pregnant UAPA patients. Taking the herein presented case into account, our literature search resulted in 16 documented cases of delivery in female UAPA patients with documented multiparity in 8 cases [19,23,27-32]. Out of these 16 women, one was admitted to hospital with an acute respiratory distress syndrome and died after delivery from a sepsis. No PH was documented. None of the 3 women with a documented $\mathrm{PH}$ died and all neonates of the 16 women were healthy. Furuya et al. documented alterations in pulmonary artery pressure (PAP) in a case series of two women with isolated UAPA and spinal anesthesia during their cesarean section [23]. Both had a normal PAP with $5 / 1 \mathrm{mmHg}$ and $8 / 4 \mathrm{mmHg}$ respective, when entering the operation room. Postpartum sympathetic stimuli were controlled by epidural anesthesia. The maximum systolic PAP was measured 2 days and 2 hours after delivery with 20 respective $22 \mathrm{mmHg}$. A similar case was reported by Koga et al., which was well controlled by spinal anesthesia [4]. These results illustrate that PAP can increase postpartum coinciding with findings of Weiss et al. and Jais et al. underlining the importance of peripartum hospitalization and PAP control $[24,26]$. In conclusion no increased risk for women with UAPA is documented in the literature. The case series of Furuya et al. may suggest a lower ability to cope increasing $\mathrm{PH}$ in case of UAPA compared to a non-high-risk pregnancy [23]. The high association of UAPA and PH emphasizes the special focus that should be put on PAP control during and after pregnancy.

\section{Conclusion}

A rare incidence and prevalence, hence potentially a reasonable number of unreported and undetected cases as well as a large variety of symptoms and symptom severity are characteristics of unilateral absence of pulmonary artery, UAPA. Therefore, there is no uniform guideline to diagnosis or therapy. To the contrary, UAPA remains a rare condition that comprises asymptomatic patients diagnosed by chance and life-threatening conditions such as acute haemorrhage or right heart failure.

Whereas revascularization procedure of the symptomatic neonate and infant can be considered as standard, there is still a therapeutic uncertainty for asymptomatic infants and adults irrespective of symptoms. The operation of an asymptomatic UAPA infant can probably prevent the later onset of major symptoms and improves quality of life, but even though in-hospital mortality rates are low $[2,9]$ there is still a considerable perioperative morbidity and longterm results or reasonable numbers are lacking so that the surgical approach will always be an individual decision. Furthermore, whether the incidental childhood UAPA will become symptomatic is not certain. Consequently, there is a particular demand of individual decision-making for physicians and parents. In the symptomatic adult, pneumonectomy can be a curative and low risk treatment approach for recurrent haemoptysis and infections [2,9]. Pregnancy and UAPA is suggested to require special care in the peripartum period although no increased mortality is documented. The high association of UAPA and $\mathrm{PH}$ lowers life expectancy. Conservative treatment options contain those of $\mathrm{PAH}$, in comparison to idiopathic $\mathrm{PH}$ operative prevention is possible. A curative operation of the adult is still experimental and often not feasible due to suspected age-related hypoplasia or regression of the affected PA, $[2,7,8]$ but if possible heart lung transplantation can be avoided.

\section{Acknowledgement}

The authors whose names are listed certify that they have No affiliations with or involvement in any organization or entity with any financial interest (such as honoraria; educational grants; participation in speakers' bureaus; membership, employment, consultancies, stock ownership, or other equity interest; and expert testimony or patent-licensing arrangements), or non-financial interest (such as personal or professional relationships, affiliations, knowledge or beliefs) in the subject matter or materials discussed in this manuscript.

\section{References}

1. Ten Harkel AD, Blom NA, Ottenkamp J (2002) Isolated unilateral absence of a pulmonary artery: A case report and review of the literature. Chest 122: 1471 1477.

2. Bockeria LA, Makhachev OA, Khiriev TK, Podzolkov VP, Zelenikin MA, et al (2015) Repair of congenital heart defects associated with single pulmonary artery. Asian Cardiovasc Thorac Ann 23: 157-163.

3. Al Jabbari O, Abu Saleh WK, Ramchandani M, Scheinin S (2016) Case report Chronic recurrent unilateral pulmonary infection: Result of congenital unilateral agenesis of pulmonary artery. Methodist Debakey Cardiovasc J 12: 48-50.

4. Koga H, Hidaka T, Miyako K, Suga N, Takahashi N (2010) Age-related clinical characteristics of isolated congenital unilateral absence of a pulmonary artery. Pediatr Cardiol 31: 1186-1190.

5. Bouros D, Pare P, Panagou P, Tsintiris K, Siafakas N (1995) The varied manifestation of pulmonary artery agenesis in adulthood. Chest 108: 670-676.

6. Griffin N, Mansfield L, Redmond KC, Dusmet M, Goldstraw P, et al. (2007) Imaging features of isolated unilateral pulmonary artery agenesis presenting in adulthood: A review of four cases. Clin Radiol 62: 238-244.

7. Kruzliak P, Syamasundar RP, Novak M, Pechanova O, Kovacova G (2013) Unilateral absence of pulmonary artery: Pathophysiology, symptoms, diagnosis and current treatment. Arch Cardiovasc Dis 106: 448-454.

8. Deutsch MA, Thieme SF, Hinterseer M, Johnson TR, Pfosser A, et al. (2010) Adult presentation of combined unilateral atresia of the right proximal pulmonary artery and left patent ductus arteriosus: Case report and embryological considerations. Int J Cardiol 141: e4-e7.

9. Atik E, Tanamati C, Kajita L, Barbero-Marcial M (2006) Isolated unilatera pulmonary artery agenesis: Evaluation of natural and long term evolution after corrective surgery. Arq Bras Cardiol 87: 423-428.

10. Shakibi JG, Rastan H, Nazarian I, Paydar M, Aryanpour I, et al. (1978) Isolated unilateral absence of the pulmonary artery. Review of the world literature and guidelines for surgical repair. Jpn Heart J 19: 439-451.

11. Reñé M, Sans J, Dominguez J, Sancho C, Valldeperas J (1995) Unilateral pulmonary artery agenesis presenting with hemoptysis: Treatment by embolization of systemic collaterals. Cardiovasc Intervent Radiol 18: 251-254.

12. Ohtsuka T, Nomori H, Watanabe K, Kaji M, Ebihara A, et al. (2006) Isolated 
Citation: Jacob FJ, Vogt FM, Stahlberg E, Oechtering TH, Anton S, et al. (2018) Isolated Unilateral Absence of Pulmonary Artery (UAPA) in the Adult: Between Simple Cough and Intensive Care. J Pulm Respir Med 8: 457. doi: 10.4172/2161-105X.1000457

Page 7 of 7

unilateral absence of a pulmonary artery treated by pneumonectomy in an adult: Report of a case. Surg Today 36: 525-527.

13. Steiropoulos $P$, Archontogeorgis $K$, Tzouvelekis A, Ntolios $P$, Chatzistefanou A, et al. (2013) Unilateral pulmonary artery agenesis: A case series. Hippokratia 17: 73-76.

14. Koryllos A, Magnet F, Karagiannidis C, Goßmann A, Brockmann M, et al. (2015) Unilateral agenesis of pulmonal artery in adults: a rare disease frequently undiagnosed. Pneumologie 69: 93-98.

15. Britton J, Sachithanandan A, Srinivasan L, Ghosh S (2011) Pneumonectomy for congenital isolated unilateral pulmonary artery agenesis. Med $\mathrm{J}$ Malaysia 66: 363-364.

16. Agca M, Bulum S, Gunen H, Tokgoz F, Yarkin T, et al. (2015) Pulmonary artery agenesis: A case series. Eurasian J Pulmonol 17: 62-66.

17. Wood ER, Truesdale AT, McDonald OB, Yuan D, Hassell A, et al. (2004) A unique structure for epidermal growth factor receptor bound to GW572016 (Lapatinib): Relationships among protein conformation, inhibitor off-rate, and receptor activity in tumor cells. Cancer Res 64: 6652-6659.

18. Heper G, Korkmaz ME (2007) High-pressure pulmonary artery aneurysm and unilateral pulmonary artery agenesis in an adult. Tex Heart Inst J 34: 425-430.

19. Shostak E, Sarwar A (2009) A 50-year-old woman with dyspnea, lower extremity edema, and volume loss of the right hemithorax. Chest 136: 628-632.

20. Komatsu Y, Hanaoka M, Ito M, Yasuo M, Urushihata K, et al. (2007) Unilatera absence of the pulmonary artery incidentally found after an episode of hemoptysis. Intern Med 46: 1805-1808.

21. Ghanbari H, Feldman D, David S, Saba S (2009) Unilateral absence of a left pulmonary artery: Successful therapeutic response to a combination of bosentan and warfarin. Circ Cardiovasc Imaging 2: e46-e48.

22. Olsson KM, Channick R (2016) Pregnancy in pulmonary arterial hypertension. Eur Respir Rev 25: 431-437.
23. Furuya T, lida R, Konishi J, Kato J, Suzuki T (2017) Anesthesia for cesarean section in a patient with isolated unilateral absence of a pulmonary artery. Brazilian J Anesthesiol 67: 85-88.

24. Weiss BM, Zemp L, Seifert B, Hess OM (1998) Outcome of pulmonary vascular disease in pregnancy: A systematic overview from 1978 through 1996. J Am Coll Cardiol 31: 1650-1657.

25. Bonnin M, Mercier FJ, Sitbon O, Roger-Christoph S, Jaïs X, et al. (2005) Severe pulmonary hypertension during pregnancy: Mode of delivery and anesthetic management of 15 consecutive cases. Anesthesiology 102: 1133-1137.

26. Jaïs X, Olsson KM, Barbera JA, Blanco I, Torbicki A, et al. (2012) Pregnancy outcomes in pulmonary arterial hypertension in the modern management era. Eur Respir J 40: 881-885.

27. Ko T, Gatz MG, Reisz GR (1990) Congenital unilateral absence of a pulmonary artery: A report of two adult cases. Am Rev Respir Dis 141: 795-798.

28. Vohra N, Alvarez M, Abramson AF, Lockwood CJ (1992) Hypoplastic pulmonary artery: An unusual entity mimicking pulmonary embolism during pregnancy. Obstet Gynecol 80: 483-485.

29. Ferrari M, Karrazi R, Lampronti G, Biasin C, Zuccali V, et al. (1997) Effect of changing position on arterial oxygenation in a patient with agenesia of the left pulmonary artery. Respiration 64: 371-374.

30. Yoshihara K, Kurabayashi T, Tsuchiya M, Terabayashi K, Serikawa T, et al (2006) Pregnancy with congenital unilateral absence of a pulmonary artery. Acta Obstet Gynecol Scand 85: 755-757.

31. Devulapally KK, Bhatt N, Allen JN (2010) A 36-year-old woman with a history of dextrocardia and dyspnea. Diagnosis: Unilateral absence of a pulmonary artery (UAPA) with right hypoplastic lung. Respiration 79: 81-84.

32. Aypak C, Yıkılkan H, Uysal Z, Görpelioğlu S (2012) Unilateral absence of the pulmonary artery incidentally found in adulthood. Case Rep Med 2012: 942074 